\title{
INTEGRAL MATRICES OF FIXED RANK
}

\author{
YONATAN R. KATZNELSON \\ (Communicated by William Adams)
}

\begin{abstract}
Asymptotic formulæ are derived for the number of $n \times m$ matrices of fixed rank $k$ with rational integral coefficients that are contained in a Euclidean ball of radius $T$ in $\mathbf{R}^{n \times m}$. It is assumed that $n \geq m>k \geq 1$ are fixed, and the asymptotics are valid as $T$ tends to infinity. The methods used are elementary.
\end{abstract}

\section{INTRODUCTION}

Consider a set of irreducible polynomials $\left\{\mathscr{F}_{1}, \ldots, \mathscr{F}_{s}\right\} \subset \mathbf{Z}\left[x_{1}, \ldots, x_{t}\right]$, and let

$$
\mathscr{V}=\left\{x \in \mathbf{C}^{d}: \mathscr{F}_{j}(x)=0, j=1, \ldots, s\right\} .
$$

It is a fundamental problem in Diophantine geometry to understand the distribution of the integral points, $\mathscr{V}(\mathbf{Z})$, on the real variety $\mathscr{V}(\mathbf{R})$ with respect to a Euclidean norm on $\mathbf{R}^{d}$. Specifically one analyzes the behavior of the counting function

$$
N(T, \mathscr{V})=|\{x \in \mathscr{V}(\mathbf{Z}):|x| \leq T\}|
$$

as $T$ grows. When the polynomials are homogeneous, one also studies the distribution of rational points on the associated projective variety. In the most general setting very little is known, and one is led to consider these problems when more structure is available. (See [DRS] for more background and related problems.)

Let $\mathbf{R}^{n \times m}$ be the space of real $n \times m$ matrices and $\mathbf{Z}^{n \times m}$ the set of integral points therein. We assume throughout that $n \geq m$. For $m>k \geq 1$ let

$$
V_{n, m, k}=\left\{A \in \mathbf{R}^{n \times m}: \operatorname{rank}(A)=k\right\} .
$$

$V_{n, m, k}$ is an affine algebraic set over $\mathbf{Q}$, defined by the homogeneous equations given by setting all $(k+1)$-dimensional minors equal to zero and requiring at least one $k$-dimensional minor to be nonzero. Here $V_{n, m, k}(Z)$ is infinite, and the counting function that we analyze is

$$
N(T ; n, m, k)=\#\left\{A \in V_{n, m, k}(\mathbf{Z}):|A|<T\right\},
$$

where $|\cdot|$ is the Euclidean norm: $|A|^{2}=\sum_{i j} a_{i j}^{2}$.

Received by the editors March 17, 1992 and, in revised form, June 12, 1992.

1991 Mathematics Subject Classification. Primary 11D41; Secondary 11H06, 11P21.

Research at MSRI supported by NSF grant DMS 8505550. 
In [Kat] this is done for the case $m=n, k=n-1$. In that case there is more freedom in the choice of the convex body in whose dilations we count the integral points. As a result it is shown there that the rational points in the associated projective set are equidistributed with respect to a naturally occurring measure.

The basic result here is

Theorem 1. For $n \geq m>k \geq 1$ and as $T$ tends to infinity:

$$
\begin{gathered}
\text { for } n>m, \quad N(T ; n, m, k)=\alpha(n, m, k) T^{n k}+O\left(T^{n k-1}\right) . \\
\text { for } n=m, \quad N(T ; n, n, k)=\beta(n, k) T^{n k} \log T+O\left(T^{n k}\right) .
\end{gathered}
$$

(The constants implied above in the error terms depend only on $n, m$, and $k$. This will be the case for all constants implied by the " $O$ " and " $\ll$ " notations, throughout this paper.)

The difference between the two cases follows from the fact that certain finite sums that occur in the computation are partial sums of Dirichlet series corresponding essentially to Koecher zeta functions (see [Ter]). In the first case the exponent is in the domain of convergence of the series, but in the second the exponent is at the first pole, giving the factor of $\log T$. The constants will be given explicitly in $\S 3$.

Note that the case $m=k$ is slightly less interesting from this perspective, since it follows from Theorem 1 that asymptotically almost all $n \times m$ integral matrices have full rank. A more interesting problem in this case is to require the $m \times m$ minors to take specific nonzero values. The asymptotics for the case $n=m=k$ and $\operatorname{det}(A)=a \neq 0$ has been resolved using analysis on homogeneous spaces of algebraic groups by Duke, Rudnick, and Sarnak [DRS]. (Thus the case here of $n=m, k=n-1$ can be thought of as resolving the case $\operatorname{det}(A)=0$.)

We remark that the defining equations for $V_{n, m, k}$ are homogeneous, so that we can study the associated projective sets. Standard methods immediately yield asymptotic formulæ for the number of rational points of bounded height in these sets, as a corollary to Theorem 1. We omit the discussion of these results.

The key observation is that if $A$ is an integral matrix of rank $k$, then there is a primitive $k$-dimensional lattice $\Lambda \subset Z^{m}$ such that the $n$ rows of $A$ lie in $\Lambda$. This leads one to consider the lattice $M(\Lambda) \subset \mathbf{Z}^{n \times m}$ of all matrices whose rows lie in some fixed $\Lambda$. It follows that

$$
V_{n, m, k}(\mathbf{Z}) \subset \bigcup_{\Lambda} M(\Lambda)
$$

where the union runs through all primitive $k$-dimensional lattices in $\mathbf{Z}^{m}$. While the inclusion is proper, since the right-hand side is $\bigcup_{j \leq k} V_{n, m, j}(\mathbf{Z})$, the two sides above are essentially equal from the point of view of the asymptotic formula. The discrepancy will contribute to the error terms above.

Since the $M(\Lambda)$ 's are lattices, we may apply classical results from the geometry of numbers to evaluate $\#\{A \in M(\Lambda):|A|<T\}$, which we then sum over an appropriate finite set of primitive lattices. This leads to expressions of the form 
$\sum_{d(\Lambda)<T^{k}} d(\Lambda)^{-n}$, where $d(\Lambda)$ is the covolume ('determinant') of $\Lambda$. These sums are the partial sums for Koecher zeta functions mentioned above.

The notation and terminology regarding lattices will be summarized and explained in the next section. In $\S 3$ we carry out the proof of Theorem 1, leaving the bounding of error terms for $\S 4$.

\section{LATTICES IN EUCLIDEAN SPACE}

We collect here the facts we require from the geometry of numbers. For more background, especially proofs of standard facts, we refer to [Cas, Sgl].

A lattice $\Lambda \subset \mathbf{R}^{m}$ is a discrete additive subgroup. $\Lambda$ has an integral basis $\left\{l_{1}, \ldots, l_{k}\right\}$ that spans $\Lambda$ over the integers, where $k \leq m$ is the dimension of $\Lambda$ and is independent of the particular choice of basis. Let $E(\Lambda)$ be the $k$-dimensional subspace that $\Lambda$ spans over $\mathbf{R}$, and then we denote by $d(\Lambda)$ the volume in $E(\Lambda)$ of a fundamental region for $\Lambda$, for example, the volume of the parallelepiped spanned by an integral basis of $\Lambda$; it is an invariant of the lattice. The following well-known formula is useful in computation and elucidates the connection with Koecher zeta functions. Let $L \in \mathbf{R}^{m \times k}$ be a matrix whose $k$ columns form an integral basis for $\Lambda$, and let ${ }^{t} L$ be its transpose. Then ${ }^{t} L L$ is a $k \times k$ symmetric matrix and

$$
d(\Lambda)=\left|\operatorname{det}\left({ }^{t} L L\right)\right|^{1 / 2} .
$$

For any basis of $\Lambda,\left\{\lambda_{1}, \ldots, \lambda_{k}\right\}$, we clearly have $d(\Lambda) \leq \prod_{j=1}^{k}\left|\lambda_{j}\right|$. From Minkowski's theory of reduction, we know that there exists a constant $c_{1}(k)>$ 0 , such that every $k$-dimensional lattice has a reduced basis satisfying [Sgl]

$$
\prod_{j=1}^{k}\left|l_{j}\right| \leq c_{1}(k) d(\Lambda) .
$$

A reduced basis is characterized by the fact that it minimizes the above product. For notational covenience, we will assume that any basis is ordered from shortest vector to longest vector.

Let $B_{T} \subset \mathbf{R}^{D}$ denote the ball of radius $T: B_{T}=\left\{x \in \mathbf{R}^{D}:|x|<T\right\}$. Denote by $v(D)$ the volume of $B_{1}$, so that $\operatorname{vol}\left(B_{T}\right)=v(D) T^{D}$. The main fact that we use from the geometry of numbers is Lemma 1 . The error term is in a slightly more precise form than that usually found in the literature, so we indicate the proof briefly. (An even more precise form may be found in [Sch].)

Lemma 1. Let $\Lambda$ be a D-dimensional lattice in $\mathbf{K}^{D}$ with reduced basis $\left\{l_{1}, \ldots\right.$, $\left.l_{D}\right\}$, satisfying $\left|l_{D}\right| \ll_{D} T$. Let $N(T, \Lambda)=\#\left(\Lambda \cap B_{T}\right)$. Then

$$
N(T, \Lambda)=\frac{v(D) T^{D}}{d(\Lambda)}+O\left(\frac{T^{D-1}}{\left|l_{1}\right|\left|l_{2}\right| \cdots\left|l_{D-1}\right|}\right)
$$

where the implied constant depends only on $D$.

Proof. The diameter of $\Lambda$ is less than $D\left|l_{D}\right|$; hence,

$$
\left|N(T, \Lambda)-\frac{v(D) T^{D}}{d(\Lambda)}\right| \leq \#(\Sigma \cap \Lambda),
$$


where $\Sigma$ is the set of points of distance no more than $D\left|l_{D}\right|$ from the boundary of $B_{T}$. Now, $\#(\Sigma \cap \Lambda) \ll_{D} \operatorname{vol}(\Sigma) / d(\Lambda)$, and $\operatorname{vol}(\Sigma) \ll_{D}\left|l_{D}\right| T^{D-1}$. The lemma now follows from (4).

In order to bound the error terms we require the following 'geometric' characterization of reduced bases. It expresses the fact that these bases are almost orthogonal. For a nonzero vector $l \in \mathbf{R}^{n}$ denote by $H(l)$ the set of vectors in $\mathbf{R}^{n}$ whose distance to $l^{\perp}$, the $(n-1)$-dimensional subspace orthogonal to $l$, is bounded by $|l| / 2$.

Proposition 2. Let $\left\{l_{1}, \ldots, l_{t}\right\}$ be a reduced basis for $\Lambda$. Then for any pair of elements therein, $l_{i}$ and $l_{j}$, with $\left|l_{j}\right| \geq\left|l_{i}\right|, l_{j} \in H\left(l_{i}\right)$.

Proof. The vector $l_{i}-l_{j}$ can replace either $l_{i}$ or $l_{j}$ in the integral basis, so that by our assumption of minimality on the basis $\left\{l_{1}, \ldots, l_{t}\right\}$ it follows that $\left|l_{i}-l_{j}\right| \geq\left|l_{j}\right|$. Denote by $\theta_{i, j}$ the (acute) angle between $l_{i}$ and $l_{j}$. From the law of cosines we conclude that $\cos \left(\theta_{i, j}\right) \leq 1 / 2\left|l_{i}\right|\left|l_{j}\right|^{-1}$. But the distance of $l_{j}$ from $l_{i}^{\perp}$ is precisely $\left|l_{j}\right| \cos \left(\theta_{i, j}\right)$.

We will be limiting our attention to sublattices of $\mathbf{Z}^{m}$. If $\Lambda \subset \mathbf{Z}^{m}$ is $k$ dimensional, we say that $\Lambda$ is primitive if it is not properly contained in any other $k$-dimensional sublattices of $\mathbf{Z}^{m}$. Let $P(X, m, k)$ denote the number of primitive $k$-dimensional lattices $\Lambda \subset \mathbf{Z}^{m}$ with $d(\Lambda)<X$.

Schmidt [Sch] proves the following

Theorem.

$$
P(X, m, k)=a(m, k) X^{m}+O\left(X^{m-b(m, k)}\right),
$$

where

$$
\begin{aligned}
a(m, k)= & \frac{1}{m}\left(\begin{array}{c}
m \\
k
\end{array}\right) \frac{v(m) v(m-1) \cdots v(m-k+1)}{v(1) v(2) \cdots v(k)} \\
& \cdot \frac{\zeta(2) \zeta(3) \cdots \zeta(k)}{\zeta(m) \zeta(m-1) \cdots \zeta(m-k+1)}
\end{aligned}
$$

(when $k=1$ the empty product of $\zeta$ 's is set equal to 1 ) and

$$
b(m, k)=\max (1 / k, 1 / m-k) .
$$

\section{Theorem 1: The MAIN TERM}

In this section, $\Lambda$ will always denote a primitive $k$-dimensional lattice in $\mathbf{Z}^{m}$. Recall that $M(\Lambda)$ is the set of all $n \times m$ matrices all of whose rows lie in $\Lambda . M(\Lambda)$ is clearly both discrete and an additive subgroup of $\mathbf{R}^{n \times m}$ and, hence, is a lattice. We construct a basis for $M(\Lambda)$ from a fixed reduced basis, $\left\{l_{1}, \ldots, l_{k}\right\}$, of $\Lambda$. Let $L_{i, j}$ denote the matrix whose $i$ th row is $l_{j}$ and all of whose other $n-1$ rows are zero. Then $\left\{L_{i, j}: 1 \leq i \leq n, 1 \leq j \leq k\right\}$ is an integral basis for $M(\Lambda)$, from which we see that $M(\Lambda)$ is $n k$-dimensional. This basis has some pleasant features. First of all $\left|L_{i, j}\right|=\left|l_{j}\right|$ for all $i$ and $j$. Furthermore, since $L_{i, j}$ and $L_{s, t}$ are properly orthogonal whenever $i \neq s$, it 
follows that

$$
d(M(\Lambda))=d(\Lambda)^{n}
$$

These two facts, together with the fact that $\left\{l_{1}, \ldots, l_{k}\right\}$ was taken to be reduced, shows that $\left\{L_{i, j}\right\}$ satisfies (4) with a constant that is no worse than the $c_{1}(k)^{n}$.

Next we need to determine the (finite) set of lattices in $\mathbf{Z}^{n}$ that actually contribute to $N(T, n, m, k)$, i.e., for which $M(\Lambda) \cap B_{T}$ contains rank $k$ matrices.

Assume that $A \in V_{n, m, k}(Z) \cap B_{T}$. Then the rows of $A$ are all bounded in length by $T$ and span a $k$-dimensional lattice in $Z^{n}$. This lattice is contained in a primitive $k$-dimensional lattice $\Lambda \subset Z^{n}$, and, since $\Lambda$ contains $k$ linearly independent vectors of length less than $T$ (the rows of $A$ ), it follows from the theory of reduction that $\Lambda$ has a reduced basis $\left\{l_{1}, \ldots, l_{k}\right\}$ with $\left|l_{k}\right| \leq c_{2}(k) T$. We say that these lattices are 'bounded by $T$ ' and denote the set of these objects by $C_{T}$. We conclude that $A \in V_{n, m, k}(\mathbf{Z}) \cap B_{T}$ implies that there exists $\Lambda \in C_{T}$ with $A \in M(\Lambda)$. Conversely, there is a constant $c_{3}(n, k)$ such that if $\Lambda \in C_{T}$ then $M(\Lambda)$ contains rank $k$ matrices bounded in norm by $c_{3}(n, k) T$. The precise values of these constants do not affect the main term, as we shall see, and hence we may take $C_{T}$ to be the correct set of lattices to consider. It follows that

$$
N(T ; n, m, k)=\sum_{\Lambda \in C_{T}} N(T, M(\Lambda))+\varepsilon_{1},
$$

where $\varepsilon_{1}$ is the error due to counting matrices of rank less than $k$. Equivalently, these are the matrices whose rows lie in the intersection of two or more distinct $\Lambda$ 's in $C_{T}$.

For $\Lambda \in C_{T}$ (with reduced basis $\left\{l_{1}, \ldots, l_{k}\right\}$ ) we may apply Lemma 1 to $M(\Lambda)$ (with corresponding basis $\left\{L_{i, j}\right\}$ ) yielding

$$
N(T, M(\Lambda))=\frac{v(n k) T^{n k}}{d(\Lambda)^{n}}+O\left(\frac{T^{n k-1}}{\left|l_{1}\right|^{n} \cdots\left|l_{k-1}\right|^{n}\left|l_{k}\right|^{n-1}}\right),
$$

where we used (7) and replaced $\left|L_{i, j}\right|$ by $\left|l_{j}\right|$ at every occurrence of the former in the denominator of the error term. Applying (8) yields

$$
N(T ; n, m, k)=v(n k) T^{n k} \sum_{\Lambda \in C_{T}} d(\Lambda)^{-n}+\varepsilon_{1}+\varepsilon_{2},
$$

where $\varepsilon_{2}$ is the sum over $C_{T}$ of the " $O$ " term in (8).

We would like to replace $C_{T}$ by a more convenient set. Note that if $\Lambda \in C_{T}$ then $d(\Lambda) \ll T^{k}$. The converse is not true, that is to say that there are 'skewed' lattices $\Lambda^{\prime}$ with reduced bases $\left\{l_{1}, \ldots, l_{k}\right\}$ satisfying $\left|l_{k}\right| \gg d\left(\Lambda^{\prime}\right)^{1 / k}$. It will be shown, however, that there are relatively few of them. With this in mind we have

$$
N(T ; n, m, k)=v(n k) T^{n k} \sum_{d(\Lambda) \ll T^{k}} d(\Lambda)^{-n}+\varepsilon_{1}+\varepsilon_{2}+\varepsilon_{3},
$$

where $\varepsilon_{3}$ is the contribution to the sum of 'skewed' lattices-lattices $\Lambda \notin C_{T}$ for which $d(\Lambda) \ll T^{k}$. Leaving the error terms for the next section, it remains 
to estimate

$$
S(T ; n, m, k)=\sum_{d(\Lambda)<T^{k}} d(\Lambda)^{-n}
$$

Summation by parts together with Schmidt's formula (6) yields

$$
S(T ; n, n, k)=n k \cdot a(n, k) \log T+O(1),
$$

while for $n>m$ we have the slightly less informative $S(T ; n, m, k)=$ const $+O\left(T^{m k-n k}\right)$. Inserting (11) in (10) gives

$$
N(T ; n, n, k)=\beta(n, k) T^{n k} \log T+O\left(T^{n k}+\varepsilon_{1}+\varepsilon_{2}+\varepsilon_{3}\right),
$$

with

$$
\begin{aligned}
\beta(n, k)= & k \cdot v(n k)\left(\begin{array}{l}
n \\
k
\end{array}\right) \frac{v(n) v(n-1) \cdots v(n-k+1)}{v(1) v(2) \cdots v(k)} \\
& \cdot \frac{\zeta(2) \zeta(3) \cdots \zeta(k)}{\zeta(n) \zeta(n-1) \cdots \zeta(n-k+1)},
\end{aligned}
$$

keeping in mind the remark that followed (6) in the case that $k=1$.

To evaluate $S(T ; n, m, k)$ when $n>m$ we describe briefly the relation to Koecher zeta functions, referring to [Ter] for any further details. For a positive symmetric matrix $X \in \mathbf{R}^{m \times m}, \Re(s)>m / 2$, and $m \geq k$ we define the Koecher zeta function

$$
Z_{k, m-k}(X, s)=\sum_{L \in \mathbf{Z}^{m \times k} / \mathrm{GL}(k, \mathrm{Z})} \operatorname{det}\left({ }^{\mathrm{t}} L X L\right)^{-s},
$$

where the summation extends over matrices $L$ of full rank $k$. The equivalence classes of rank $k$ matrices in $\mathbf{Z}^{m \times k} / \mathrm{GL}(k, \mathbf{Z})$ are in 1-1 correspondence with the set of all $k$-dimensional lattices $\Delta \subset Z^{m}$, since the columns of such a matrix span a $k$-dimensional lattice, and two such matrices span the same lattice iff they are equivalent mod $\mathrm{GL}(k, \mathbf{Z})$. Summing over all $k$-dimensional lattices, we have from (3) that

$$
Z_{k, m-k}(I, s)=\sum_{\Delta \subset \mathbf{Z}^{m}} d(\Delta)^{-2 s}
$$

where $I$ is the identity matrix. From [Ter, $§ 4.4$, Lemma 6] (decomposition of integral $m \times k$ rank $k$ matrices), it follows that

$$
Z_{k, m-k}(I, s)=Z_{k, 0}(I, s) \sum_{\Lambda \subset Z^{m}} d(\Lambda)^{-2 s},
$$

where the summation now extends over primitive lattices. Terras shows that

$$
Z_{k, 0}(I, s)=\zeta(2 s) \zeta(2 s-1) \cdots \zeta(2 s-k+1) .
$$

Hence, for $n>m$

$$
S(T ; n, m, k)=\frac{Z_{k, m-k}(I, n / 2)}{\zeta(n) \zeta(n-1) \cdots \zeta(n-k+1)}+O\left(T^{-k}\right),
$$

from which it follows that

$$
N(T ; n, m, k)=\alpha(n, m, k) T^{n k}+O\left(T^{m k}+\varepsilon_{1}+\varepsilon_{2}+\varepsilon_{3}\right),
$$


with

$$
\alpha(n, m, k)=\frac{v(n k) Z_{k, m-k}(I, n / 2)}{\zeta(n) \zeta(n-1) \cdots \zeta(n-k+1)}
$$

\section{ERROR TERMS}

In this section $\Lambda$ will once again denote a primitive $k$-dimensional lattice in $\mathbf{Z}^{m}$, and $\Lambda_{1}$ will denote a primitive $(k-1)$-dimensional lattice. We fix $n \geq m>k$ and distinguish between $n=m$ and $n>m$ where necessary.

Throughout this section we will be bounding sums of the form $\sum|l|^{-h}$, ranging over the integer points in certain bounded domains in $\mathbf{R}^{n}$. To do this we apply the following observation. Assume that $D \subset \mathbf{R}^{m}$ satisfies the 'growth condition'

$$
\#\left(D \cap \mathbf{Z}^{m} \cap B_{X}\right) \ll X^{h_{1}}
$$

where the implied constant depends only on $m$. Set

$$
\varphi_{D}(n)=\#\left\{l \in D \cap \mathbf{Z}^{m}: n \leq|l|<n+1\right\} .
$$

Then applying summation by parts to

$$
\sum_{a \leq n<b} \varphi_{D}(n) n^{-h_{2}}
$$

yields

$$
\sum_{\substack{a \leq|l|<b \\ l \in D}}|l|^{-h_{2}} \ll a^{h_{1}-h_{2}}+b^{h_{1}-h_{2}}+\int_{a}^{b} t^{h_{1}-h_{2}-1} d t,
$$

where the implied constant depends on $D, h_{1}$, and $h_{2}$.

We will be applying (13) to three basic domains: $\mathbf{R}^{m}, H\left(l_{i}\right)$, and $H\left(l_{i}\right) \cap$ $H\left(l_{j}\right)$, where $l_{i}$ and $l_{j}$ are elements of a reduced basis for some lattice (recall the definition preceding Proposition 2). We require estimates for the number of $\mathrm{Z}^{m}$ points contained in the intersection of these domains with $B_{X}$. In all three cases the volume of the intersection provides the correct order of magnitude, by Lemma 1 and the observation that for all three of these the $m$-dimensional volume $\gg$ the $(m-1)$-dimensional volume of the boundary. The volume of $\mathbf{R}^{m} \cap B_{X}$ is $O\left(X^{m}\right)$. The volume of $H\left(l_{i}\right) \cap B_{X}$ is $O\left(\left|l_{i}\right| X^{m-1}\right)$, and, using the fact that $l_{j} \in H\left(l_{i}\right)$, the volume of $H\left(l_{i}\right) \cap H\left(l_{j}\right) \cap B_{X}$ is $O\left(\left|l_{i}\right|\left|l_{j}\right| X^{m-2}\right)$.

The first error, $\varepsilon_{1}$, is due to overcounting matrices that appear in the intersection of two or more $M(\Lambda)$ 's. Such a matrix $A$ belongs to $M\left(\Lambda_{1}\right)$, for some ( $k-1$ )-dimensional $\Lambda_{1}$. (If $k=1$ there is nothing to prove here.) It follows that

$$
\varepsilon_{1} \ll \sum_{\Lambda_{1} \in C_{T}} n\left(\Lambda_{1}, T\right) N\left(T, M\left(\Lambda_{1}\right)\right),
$$

where $n\left(\Lambda_{1}, T\right)$ is the number of $k$-dimensional $\Lambda$ 's in $C_{T}$ properly containing $\Lambda_{1}$. (We are using $C_{T}$ generically for all dimensions, where it is understood that the constant implied in the definition changes with the dimension.)

If $\Lambda$ and $\Lambda^{\prime}$ are two primitive $k$-dimensional lattices in $C_{T}$, both containing $\Lambda_{1}$, then it follows that there are two primitive integral vectors $l$ and $l^{\prime}$ such that $\Lambda=\Lambda_{1} \oplus \mathbf{Z} \cdot l$ and $\Lambda^{\prime}=\Lambda_{1} \oplus \mathbf{Z} \cdot l^{\prime}$. Furthermore, $|l|,\left|l^{\prime}\right| \ll T$. Now, 
$\Lambda=\Lambda^{\prime}$ iff $l-l^{\prime} \in \Lambda_{1}$, so that $n\left(\Lambda_{1}, T\right)$ is bounded by the number of $\Lambda_{1}$ equivalence classes in $Z^{m} \cap B_{T}$. Such an equivalence class is just a shift of $\Lambda_{1}$, and so there are constants $e_{2} \geq e_{1}>0$, depending only on $k$, such that the number of elements in such a class satisfies

$$
e_{1} \frac{T^{k-1}}{d\left(\Lambda_{1}\right)} \leq \#\left(\left(\Lambda_{1}+l\right) \cap B_{T}\right) \leq e_{2} \frac{T^{k-1}}{d\left(\Lambda_{1}\right)} .
$$

The factor of $T^{k-1} / d\left(\Lambda_{1}\right)$ is valid by Lemma 1 since $\Lambda_{1} \in C_{T}$. The total number of points in $B_{T}$ is $O\left(T^{m}\right)$, and hence

$$
n\left(\Lambda_{1}, T\right)=O\left(T^{m-k+1} d\left(\Lambda_{1}\right)\right) .
$$

Recalling that $N\left(T, M\left(\Lambda_{1}\right)\right)=O\left(T^{n k-n} / d\left(\Lambda_{1}\right)^{n}\right)$, we have

$$
\varepsilon_{1} \ll T^{n k-n+m-k+1} \sum_{\Lambda_{1} \in C_{T}} d\left(\Lambda_{1}\right)^{1-n} .
$$

We replace the condition $\Lambda_{1} \in C_{T}$ by $d\left(\Lambda_{1}\right)<T^{k-1}$, as in $\S 3$, and apply summation by parts. When $n>m$ the sum gives us a factor no larger than $O(\log T)$, while the factor outside the sum is no larger than $T^{n k-2}$ (since we assume here that $k>1$ ). Hence, for this case $\varepsilon_{1} \ll T^{n k-2} \log T$. When $n=m$ the sum contributes a factor of $T^{k-1}$, so that $\varepsilon_{1} \ll T^{n k}$. In either case, $\varepsilon_{1}$ is of the size promised or smaller.

Recall that

$$
\varepsilon_{2} \ll T^{n k-1} \sum_{\Lambda \in C_{T}}\left|l_{1}\right|^{-n} \cdots\left|l_{k-1}\right|^{-n}\left|l_{k}\right|^{1-n},
$$

where $\left\{l_{1}, \ldots, l_{k}\right\}$ is a reduced basis for $\Lambda$, ordered from shortest to longest. Applying Proposition 2 we have

$$
\varepsilon_{2} \ll T^{n k-1} \sum_{1<\left|l_{1}\right| \ll T}\left|l_{1}\right|^{-n} \sum_{\substack{\left|l_{1}\right| \leq\left|l_{2}\right|<T \\ l_{2} \in H\left(l_{1}\right)}}\left|l_{2}\right|^{-n} \ldots \sum_{\substack{\left|l_{k}\right| 1 \leq\left|l_{k}\right| \leq T \\ l_{k} \in H\left(l_{1}\right) \cap H\left(l_{k-1}\right)}}\left|l_{k}\right|^{1-n} .
$$

By (13) the innermost sum is $O\left(\left|l_{1}\right|\right)$ when $n=m$ and $O\left(\left|l_{1}\right| /\left|l_{k-1}\right|\right)$ when $n>m$. The next sum is

$$
\sum_{\substack{\left|l_{k-2}\right| \leq|\leq| l_{k-1} \mid \leq T \\ l_{k-1} \in H\left(l_{k-2}\right)}}\left|l_{k-1}\right|^{-h}
$$

where $h=n$ when $n=m$ and $h=n+1$ otherwise, so that this sum is $O(1)$ in the first case and $O\left(\left|l_{k-2}\right|^{-1}\right)$ in the latter case. Continuing in this manner we obtain that multiple sum contributes a factor of $O(T)$ when $n=m$ and a factor of $O(1)$ otherwise. Hence, $\varepsilon_{2}=O\left(T^{n k}\right)$ when $n=m$ and $\varepsilon_{2}=O\left(T^{n k-1}\right)$ otherwise.

The last error term comes from the contribution to (10) of skewed lattices. We replace the term $d(\Lambda)^{-n}$ by the product $\left|l_{1}\right|^{-n} \cdots\left|l_{k}\right|^{-n}$ (by (4)) and sum over sets of vectors satisfying Proposition 2 , and the conditions $\left|l_{1}\right|<T$ and $T<\left|l_{k}\right|$, to account for the skewedness of the lattices that contribute this error. 
We have

$$
\varepsilon_{3} \ll T^{n k} \sum_{1 \leq\left|l_{1}\right|<T}\left|l_{1}\right|^{-n} \sum_{\substack{\left|l_{1}\right| \leq\left|l_{2}\right| \\ l_{2} \in H\left(l_{1}\right)}}\left|l_{2}\right|^{-n} \cdots \sum_{\substack{T<\left|l_{k}\right| \\ l_{k} \in H\left(l_{1}\right)}}\left|l_{k}\right|^{-n} .
$$

The innermost sum contributes a factor which is $O\left(\left|l_{1}\right| / T\right)$. The next sum is

$$
\sum_{\substack{\left|l_{k-2}\right|<\left|l_{k-1}\right| \\ l_{k-1} \in H\left(l_{k-2}\right)}}\left|l_{k-1}\right|^{-n}
$$

which is $O(1)$ when $n=m$ and $O\left(\left|l_{k-2}\right|^{-1}\right)$ otherwise. Continuing in this manner we conclude, as with $\varepsilon_{2}$, that $\varepsilon_{3}=O\left(T^{n k}\right)$ when $n=m$ and $\varepsilon_{3}=$ $O\left(T^{n k-1}\right)$ otherwise.

\section{ACKNOWLEDGMENTS}

The author wishes to thank the referee and Professor W. Schmidt for their helpful comments and suggestions.

\section{REFERENCES}

[Cas] J. W. S. Cassels, An introduction to the geometry of numbers, Springer-Verlag, New York, 1971.

[DRS] W. Duke, Z. Rudnick, and P. Sarnak, Density of integer points on affine homogeneous varieties, Duke Math J. (to appear).

[Kat] Y. Katznelson, Singular matrices and a uniform bound for congruence groups of $S L_{n}(\mathbf{Z})$, Duke Math. J. 69 (1993), 121-136.

[Sch] W. Schmidt, Asymptotic formulae for point lattices of bounded determinant and subspaces of bounded height, Duke Math J. 35 (1968), 327-339.

[Sgl] C. L. Siegel, Lectures on the geometry of numbers, Springer-Verlag, New York, 1988.

[Ter] A. Terras, Harmonic analysis on symmetric spaces and applications. II, Springer-Verlag, New York, 1988.

Mathematical Sciences Research Institute, 1000 Centennial Drive, Berkeley, CaliFORNIA 94720

Current address: Department of Mathematics, University of Maryland, College Park, Maryland 20742 